\title{
Vaccine containing immunologic adjuvants with a wide range of activity to provide protection against COVID-19
}

Author: Mulugeta Berhanu, Ethiopian Agricultural Transformation Agency

Email: mulugetach100@gmail.com; Tel:+251921433836

\begin{abstract}
COVID-19 is a devastating viral disease which can be prevented by vaccination. Immunologic adjuvants are key to develop an effective vaccines against infectious diseases in human and nonhuman primates. Vaccines containing an appropriate adjuvants are crucial for the prevention of various infectious diseases which are the biggest threats to human and animal health. This paper proposes a wide spectrum immunologic adjuvant for vaccine development against COVID-19 which is the current global problem. It has been reported that a wide range of immune cells are involved in the body's response to SARS CoV2 infection. Therefore, vaccine with a widespectrum immunologic adjuvant can be used to provide protection against COVID-19. Lack of adjuvants that can induce the required immune responses is a serious impediment to vaccine development against this devastating virus. The approved adjuvants such as aluminum salts and MF59 exhibit a narrow range of activity. In an attempt to solve this problem, it is crucial to develop new adjuvants which can trigger a wide range of immune cells.
\end{abstract}

Key words: Adjuvants for COVID-19 vaccines, Aluminum salts, COVID-19, Immunologic adjuvants, MF-59, SARS CoV2, Vaccine development against COVID-19, Vaccine with a widespectrum immunologic adjuvant 


\section{Introduction}

Vaccine has played a critical role in protecting the global community from various infectious diseases. Vaccine development against COVID-19 is hindered by lack of approved adjuvants that can induce the desired immune responses. The approved adjuvants such as aluminum salts and MF59 exhibit a narrow range of activity. To circumvent this problem, it is crucial to develop a wide spectrum immunologic adjuvant that can be used in vaccine against COVID-19.

Discovery of new adjuvants with a wide range of activity is necessary to develop an effective vaccine against the novel corona virus. Immunologic adjuvants are key to develop an effective vaccines against infectious diseases in human and non-human primates. Adjuvants are immunostimulatory molecules that enhances immune response to vaccine antigens. Immunologic adjuvants are co-administered with a specific antigen to trigger rapid and robust humoral and cellular immune responses. Using antigen in combination with adjuvant offers significant advantage over using antigen alone because adjuvant improve immunogenicity of antigen.

Comprehensive understanding of immune mechanisms needed for protection against infectious diseases played a critical role in developing many vaccines that we are using today. Thus, in-depth understanding of immune mechanisms required for protection against COVID-19 can help us to use better adjuvants for vaccine development against COVID-19. It has been proved that a wide range of immune cells are involved in the body's response to SARS CoV2 infection. Adjuvants with ability to induce immune cells which are involved in SARS CoV2 infection are needed to develop an effective vaccines against COVID-19.

Vaccines containing an appropriate adjuvants are important for the prevention of various infectious diseases which are the biggest threats to human and animal health. It is crucial to use adjuvants with minimal side effects and high potency so as to develop safe and efficacious vaccines. Potent adjuvants with undesirable effects are not recommended to be used in vaccine development. 


\section{Vaccine adjuvants}

Adjuvant is a term coined from the Latin word adjuvare, which means to help or to augment. Immunological adjuvants are agents that enhance specific immune responses to vaccines [15]. Aluminum hydroxide typically referred as "alum" and other aluminum salts are the most widely used adjuvants in human and animal vaccines [7]. Although vaccine adjuvants have been used for almost a century, alum is the only adjuvant licensed by the US FDA for human vaccine use [11].

Adjuvants are chemicals, proteins or derivatives of microbial formulated with vaccines to enhance innate and adaptive immune response to vaccine antigens by a variety of mechanisms [6]. There are several types of adjuvants with differing modes of action. These include mineral salts, oil emulsions, immune stimulating complexes (ISCOM), bacterial derivatives, carbohydrate adjuvants, liposomes, cytokines, virus like particles and polymeric microparticle adjuvants [12]. Nonreplicating, purified subunit or synthetic viral vaccines of the future are likely to be weak immunogens that will require immunopotentiation if they are to be effective. These marginal vaccines could be improved by combination with potent and safe immunologic adjuvants [5].

The success of adjuvants in enhancing the immune response to recombinant antigens has led many researchers to re-focus their vaccine development programs [9]. Adjuvanted vaccines afford invaluable protection against disease, and the molecular and cellular changes they induce offer direct insight into human immunobiology [14].

\section{Developing a novel immunologic adjuvant for use in vaccine against COVID-19}

The development of novel immune adjuvants is emerging as a significant area of vaccine delivery based on the continued necessity to amplify immune responses to a wide array of new antigens that are poorly immunogenic [8]. Over the past 10-15 years, a large research effort has attempted to find novel adjuvants with ability to induce a broad range of immune responses, including cellmediated immunity [13]. Recent advance in vaccine adjuvants is timely, given the need to create novel vaccines to deal with the COVID-19 pandemic [3]. 
Discovering new adjuvant that are essential components of vaccine formulations helps the development of more potent vaccine, which induce strong immune response. [6]. Vaccines containing novel adjuvant formulations are increasingly reaching advanced development and licensing stages, providing new tools to fill previously unmet clinical needs [10].

Development of new vaccine adjuvants has entered a golden age of research and development [4]. Despite the impressive success of currently approved adjuvants for generating immunity to viral and bacterial infections, there remains a need for improved adjuvants that enhance protective antibody responses, especially in populations that respond poorly to current vaccines [1]. Currently, many new vaccines are under development and there is a desire to simplify vaccination schedules both by increasing the number of components per vaccine and decreasing the number of doses required for a vaccine course. New, more effective adjuvants will be required to achieve this [2].

\section{Conclusions}

Vaccine containing an appropriate adjuvant is important to control challenging pathogens that can cause global pandemics. Vaccine with a wide spectrum immunologic adjuvant can be used to provide protection against the novel Corona virus. Therefore, it is crucial to develop novel adjuvants with a wide range of activity.

\section{Funding information}

No grant or funding has been received for conducting this study.

\section{Conflict of interest declaration}

The author declare that there is no conflict of interest. 


\section{References}

1. Coffman RL, Sher A, Seder RA. Vaccine adjuvants: putting innate immunity to work. Immunity. 2010 Oct 29;33(4):492-503. doi: 10.1016/j.immuni.2010.10.002. PMID: 21029960; PMCID: PMC3420356.

2. Cox JC, Coulter AR. Adjuvants--a classification and review of their modes of action. Vaccine. 1997 Feb;15(3):248-56. doi: 10.1016/s0264-410x(96)00183-1. PMID: 9139482.

3. Derek T. O'Hagan, Rushit N. Lodaya, Giuseppe Lofano (2020): The continued advance of vaccine adjuvants- 'we can work it out' https://doi.org/10.1016/j.smim.2020.101426 4. Didierlaurent, A. M. et al. AS04, an aluminum salt- and TLR4 agonist-based adjuvant system, induces a transient localized innate immune response leading to enhanced adaptive immunity. $J$. Immunol. 183, 6186-6197 (2009).

5. Edelman R. Vaccine adjuvants. Rev Infect Dis. 1980 May-Jun;2(3):370-83. doi: 10.1093/clinids/2.3.370. PMID: 6997966.

6. Getu Ayele, 2020 Department of Veterinary Microbiology, College of Veterinary Medicine and Agriculture, University in Addis Ababa, Bishoftu, Ethiopia : Review on recent advances of vaccine adjuvants

7. Lindblad EB. Aluminium compounds for use in vaccines. Immunol Cell Biol. 2004;82:497505 .

8. Oyewumi MO, Kumar A, Cui Z. Nano-microparticles as immune adjuvants: correlating particle sizes and the resultant immune responses. Expert Rev Vaccines. 2010;9(9):1095-1107. doi:10.1586/erv.10.89

9. Reed SG, Bertholet S, Coler RN, Friede M. New horizons in adjuvants for vaccine development. Trends Immunol. 2009 Jan;30(1):23-32. doi: 10.1016/j.it.2008.09.006. Epub 2008 Dec 6. PMID: 19059004.

10. Reed, S., Orr, M. \& Fox, C. Key roles of adjuvants in modern vaccines. Nat Med 19, 15971608 (2013). https://doi.org/10.1038/nm.3409

11. Schmidt CS, Morrow WJ, Sheikh NA. Smart adjuvants. Expert Rev Vaccines. 2007 Jun;6(3):391-400. doi: 10.1586/14760584.6.3.391. PMID: 17542754.

12. Sivakumar SM, Safhi MM, Kannadasan M, Sukumaran N. Vaccine adjuvants - Current status and prospects on controlled release adjuvancity. Saudi Pharm J. 2011 Oct;19(4):197-206. doi: 10.1016/j.jsps.2011.06.003. Epub 2011 Jun 25. PMID: 23960760; PMCID: PMC3744968. 
13. Sjolander et al., 1998 A. Sjolander, C. John Cox, G. Ian Barr ISCOMs: an adjuvant with multiple functions J. Leukoc. Biol., 64 (1998), pp. 713-723

14. Sobolev O, Binda E, O'Farrell S, Lorenc A, Pradines J, Huang Y, Duffner J, Schulz R, Cason J, Zambon M, Malim MH, Peakman M, Cope A, Capila I, Kaundinya GV, Hayday AC. Adjuvanted influenza-H1N1 vaccination reveals lymphoid signatures of age-dependent early responses and of clinical adverse events. Nat Immunol. 2016 Feb;17(2):204-13. doi: 10.1038/ni.3328. Epub 2016 Jan 4. Erratum in: Nat Immunol. 2016 Apr;17(4):469. PMID: 26726811; PMCID: PMC6485475.

15. Vogel, 1998 F.R. Vogel Adjuvants in perspective F. Brown, L.R. Haaheim (Eds.), Modulation of the Immune Response to Vaccine Antigens. Dev. Biol. Stand., vol. 92, Karger, Basel (1998), pp. 241-248 